\title{
SOME NEW POINTS IN THE ANATOMY OF THE NASAL SEPTUM, AND THEIR SURGICAL SIGNIFICANCE. ${ }^{1}$
}

\author{
By J.. L. Aymard, Captain, R.A.M.C. (Temp.), \\ Surgeon to the Ear, Nose, and Throat Department, The Queen's Hospital for \\ Facial Injuries, Sidcup.
}

In recent years more particularly, the progress of the anatomy of a part appears to advance concurrently with its surgical or medical importance, and the aratomy of the nasal cavities is no exception.

The existence of two distinct movable joints in the septum does not appear to have been recognised as such, and the distribution of the perichondrium cannot be said to have been either described by the anatomist or understood by the surgeon.

The presence of a permanent vascular arrangement within the septal cartilage has apparently also been overlooked.

The septal cartilage is usually represented as being a perpendicular plate attached behind, below, and above, and is illustrated as presenting a free border covered only by the soft parts extending from the anterior border of the nasal bones forwards and downwards, and embedded between the median borders of the lateral cartilages. In the first place, exception must be taken to the designation of the upper lateral cartilages. They never exist as separate cartilages, neither are they separable except by force from the rest of the septal cartilage. Would it not be far more correct to describe each so-called lateral cartilage as the lamina triangularis, and the vertical cartilage as the lamina perpendicularis of one large cartilage, say the cartilago nasalis major? Prof. Macalister has already described them as the "lateral expansions of the septal cartilage."

\section{Cartilago nasalis major.}

For practical purposes it would be much better to designate landmarks indicating the general position and extent of these cartilages, rather than give dimensions which vary so considerably in each individual case.

The lamina perpendicularis (L.P., Fig. 1) of this cartilage presents four distinct borders.

The posterior border is firmly attached to the anterior edge of the lamina perpendicularis ethmoidalis (L.P.E.), the perichondrium of the former being directly continuous with the periostenm covering the latter, thus forming a strong ligamentous band.

The superior border between $i$ and ii, Fig. 1, and A, Fig. 2, is flattened and grooved to receive the prominence caused by the median junction at the under surface of the nasal bones; the groove is continued forwards, where it widens out to form the lamina triangularis (L.T., Figs. 1 and 2) or so-called lateral cartilages. This plate L.T. is triangular in shape, its base being attached to the anterior edges of the nasal bones and its apex (Fig. 1, iii) extending to the superior angle of the anterior free border. In flat noses the lamina triangularis takes a gentle curve, and in sharp narrow ones a shape corresponding.

The anterior border (Fig. 1, iv) is free and rounded, and can be readily seen through its coverings when the columna is held aside.

\section{${ }^{1}$ Reprinted from the Journal of Anatomy, April, 1917.}


The inferior border is pear-shaped in section (Fig. 1, vi ; also Fig. 2, C) and more oval (Fig. 1, vii, and Fig. 2, B). Where it forms the chondro-maxillary joint with the crista incisiva (Fig. 1, v, and Fig. 2, D) it is flattened and terminates in an obtuse angle.

Surface Markings for Localising the Posterior Border of the Lamina Perpendicularis. - The posterior border of this lamina lies a third of an inch behind the anterior edge of the nasal bones, from which it slopes irregularly downwards and backwards.

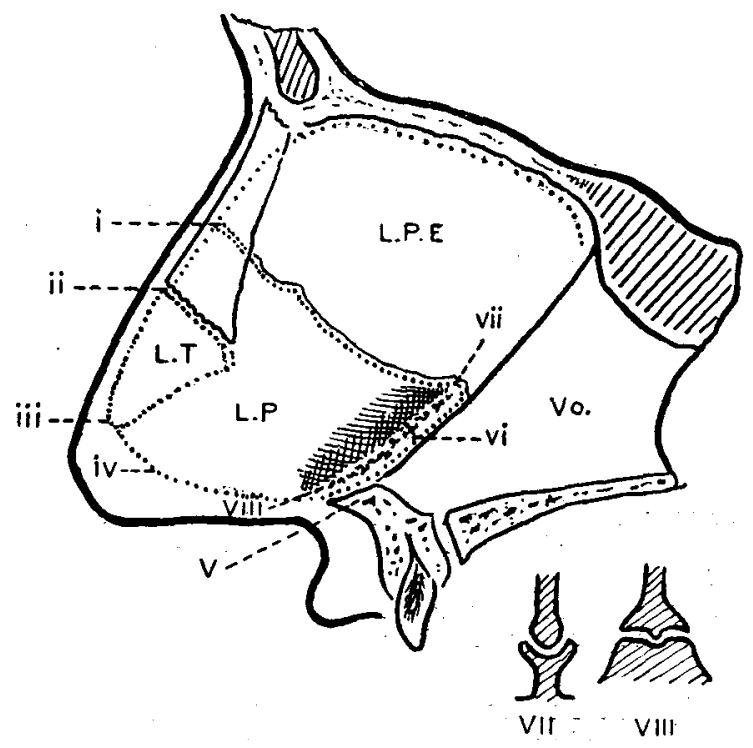

Fra. 1.-Septum of the nose showing the relationships of the septal cartilage.

VII, section across chondro-vomeral joint; VIII, section across chondro-maxillary joint.

\section{The Chondro-Vomeral and Chondro-Maxiluary Joints.}

The descriptions given in the leading works of anatomy merely state that the rounded end of the septal cartilage is received into the groove upon the anterior superior edge of the vomer and maxilla. This is correct as far as it goes, but at the same time they offer no proofs that this is not a mere osteo-cartilaginous junction.

The movement permissible at the lower septal joints forms a complete semicircle limited to a right angle each side of the perpendicular.

This, I think, is the only joint in the body permitting such extensive movements, and it appears to be constructed for the purpose. Further, it is, I believe, the only freely movable joint entirely composed of cartilage on the one hand, and a non-cartilage-covered bone on the other. A study of the joint demonstrates the fact that pressure upon the anterior superior surface of the septal cartilage causes it to bend in the direction of its long axis. Pressure upon the anterior inferior border causes the cartilage to bend obliquely upon itself, with a certain amount 
of transverse movement across the chondro-maxillary joint, combined with the lateral movement described.

Were it not for this joint, fractures and dislocations of the cartilage

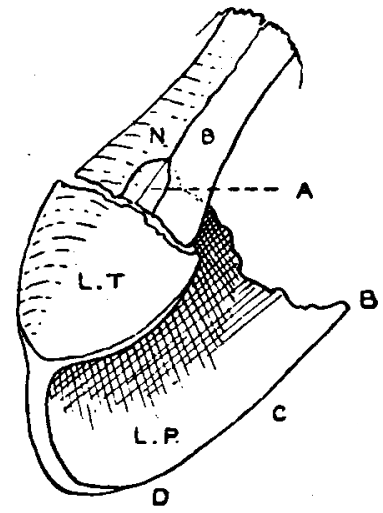

Fia. 2.-The parts of the septal cartilage. (For explanation see text.)
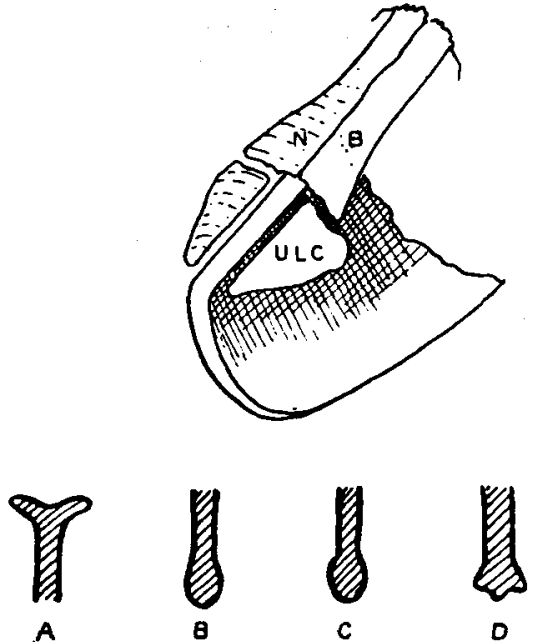

Fra. 3.-The parts of the septal cartilage as described in accepted works.

A, B, C, D, sections acrose the margin of the septal cartilage at the points indicated in Fig. 2 by corresponding letters.

would obviously be far more frequent than they are. There would be no object in a joint of a similar description with the ethmoid plate, therefore it does not exist.

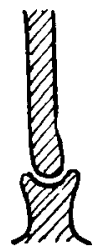

Fia. 4.

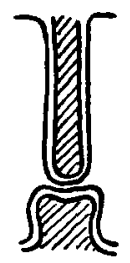

Fig. 5.

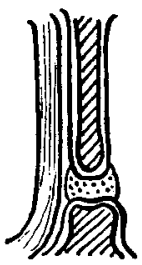

Fig. 6.

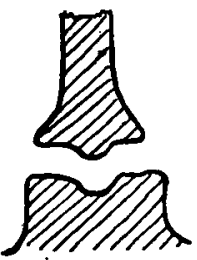

Fig. 7.

FIG. 4.-Transverse section of chondro-vomeral joint, with covering membranes removed.

F'IG. 5.-Showing the disposition of perichondrium and periosteum at chondro. vomeral joint.

Fra. 6.-The same joint showing included fat and the covering of mucous membrane on one side.

Fit. 7.-The articulating surfaces of the chondro-maxillary joint.

The Chondro-vomeral Joint (Figs. 4 and 5), as I prefer to term it, is in every sense of the word a very perfect joint constructed for a definite object. The perichondrium and periosteum are in a sense continuous, but there is much more than this to be noted.

In the first place, whatever the condition of the vomeral groove may 
be-that is to say, though the edges may be irregular or even absent here and there-the pear-shaped lower portion of the cartilage remains fairly constant and the capsular attaichments likewise. The definite object of this joint is, without doubt, to allow of free lateral movement when from any cause pressure is applied from above.

A concussion plate is also provided in the shape of a layer of fat (Fig. 6) between the periosteal perichondrial layers. The amount of movement possible, owing to the loose attachment of the perichondrium and periosteum, accounts for the fact that in a deviated septum the articular portion of the cartilage may easily occupy a transverse position across the groove (Fig. 10), forming a projection not infrequently mistaken for a crest.

The Chondro-maxillary Joint.-The portion of the inferior border of the septal cartilage entering into the formation of this joint is usually represented by the anterior third. The cartilage is flattened and pointed (Fig. 7) to rest upon the corresponding junction of the maxillary bones known as the crista incisiva. If this crest is rounded or otherwise shaped the cartilage takes a corresponding form. The corresponding coverings are similar to those of the chondro-vomeral joint, but much tougher, thicker, and looser.

The apparent object here is to allow lateral rotation as well as a diagonal lock-gate motion.

The surgical importance of the above is that a blow directed upon the anterior edge of the cartilage bends it vertically upon itself, allowing a position to be taken diagonal to the crista incisiva and thus avoiding fracture. Another point is that, unless the membranous disposition and its nature in this area of the septum is thoroughly understood, the surgeon will find himself in difficulties.

The structures referred to should always be divided carefully with a blunt-pointed bistoury.

\section{The Immediate Joint Coverings.}

It is generally taught that the perichondrium covering the cartilage and the periosteum covering the vomer are continuous and attached along the crest. Only to a certain extent is this correct. The perichondrial arrangement is somewhat complex.

In the first place, careful investigation shows that the perichondrium does not, as the usual description implies, merely merge into the periosteum. Below the orifices of the chondro-vascular system (Fig. 1, vi) the perichondrium is continued loosely around the cartilage, and is continuous with the perichondrium of the opposite surface (Fig. 5).

The periosteum (Fig. 9), on the other hand, instead of being continuous with the perichondrium, as generally described, merely sends a process upwards to be attached to the perichondrium below the vascular openings, thus loosely covering the vomeral trough (sulcus vomeralis). The perichondrium in a similar manner sends a process downwards to join the periosteum, thus forming a joint ligament.

\section{The Surgical Significance of the Points referred to.}

The anatomy of the septal cartilage is principally interesting from the point of view of its partial removal, and here I must protest against the teachings in our leading text-books. Most nasal surgeons. will agree 
with me that at least 75 per cent. of the cases presenting themselves for obstruction require the removal of only a moderate amount of cartilage and bone. The illustrations referred to advise an eighth of an inch of the anterior pillar of the septum to be left, whereas no experienced surgeon would think of leaving less than a quarter of an inch as a general rule. Further, the removal of the foundation of even this support by chiselling the maxillary crest is recommended freely without reserve and without any warning as to possible ultimate cosmetic effects. In the above case, we should teach the student not to remove a given area, but only just so much cartilage or bone as will give the necessary space for free passage of air or any other purpose.

Most surgeons use Ballenger's knife to remove cartilage, an ingenious but by no means ideal instrument. The usual procedure is to pass the knife along the upper edge of the area to be removed, down the back, and along the lower border. A reference to Figs. 10 and 11 shows the difficulty (with the cartilage lying over, and particularly with three sides cut through) of cutting the cartilage close to the bone-since any downward pressure of the knife only further depresses the free cartilage. The point to remember is, that the piece of cartilage is left possibly lying across the vomer. It being mechanically impossible to remove this portion of the cartilage with Ballenger's knife unless the sides of sulcus vomeralis are absent, I prefer to cut first the front, top, and back, and then remove the lower margin with a small, trough-shaped gouge, having, if necessary, first divided the perichondrium with a blunt bistoury.

I would draw attention to the fact that the vascular system extends to the inferior angle of the antero-inferior margin of the cartilage, and that this blood-supply is not likely, to be re-established if once cut off, which is of importance with reference to the pillar of support proposed to be left.

\section{The Septal Coverings.}

The perichondrium is very firmly attached to both surfaces of the cartilage, whereas the periosteum is only loosely attached to the bone and readily removed.

This attachment is marked everywhere except within the joint, and is due to the perichondrial processes which extend deep into the cartilage, so that when the membrane is removed these processes are for the most part torn and remain behind.

The periosteum and perichondrium are about equally adherent to the mucous membrane. Over the chondro-ethmoidal junction there is no special attachment, the one membrane merging into the other. Although the perichondrium is described as being divisible into many layers by mechanical means, I have been able to separate two layers.

The mucous membrane covering the septum has been fully described in standard works. I recognise three layers-a superficial, a middle, and an inner layer; between the two latter run the large vessels and nerves. The mucous membrane is closely attached to the perichondrium and periosteum except over the anterior third of the cartilage. In this position the membrane is very loosely attached. This fact should be noted, because it is entirely due to this loose condition in this region that failure so often takes place.

The usual incision for resection is made the full length required at 
the start, and cuts down to the cartilage, severing the perichondrium. The difficulty of endeavouring to separate the (in this region) tough mucous membrane from the closely-attached perichondrium is the cause of operative trouble.

It is far better not to incise the perichondrium at first and only to make a sufficient cut to allow the introduction of the elevator used on Watkins Williams' principle. The incision can be enlarged to the full length when the membrane around is free for a good start.

Experts teach that the cartilage be now cut through and the elevator passed in between the same and the perichondrium and raised to the full extent. As a matter of fact, I think this procedure is more apt to take place by accident than design.

I would draw the attention of students to some otber special points of importance. In the first place, all serious hæmorrhage can be avoided when operating if the positions of the larger trunks of vessels are remembered.

Just inside the anterior choana, behind its lower internal border, the palatine arteries must be carefully noted, especially when preparing to remove a portion of the crest.

The next group of vessels likely to be injured are those coursing along the yomer in the mucous membrane and in the region of its crest.

Should the mucous membrane be separated into layers (a not uncommon occurrence), it follows that in using the punch forceps these vessels are very likely to be divided between the bone and the punch. The palatine nerve may also be destroyed in a similar manner, thus leading to subsequent trophic changes in the mucous membrane.

The Significance of the Perichondrial Attachment in relation to the Operation of Sub-Mucous Resection.

In all the leading works upon this subject, this operation is primarily described as a muco-perichondrial detachment. I say primarily, because in one illustration the sub-mucous resection is referred to, and upon the same page it is described as muco-perichondrial, thus greatly confusing the student. It is clearly wrong to describe the operation as a sub-mucous perichondrial one. The illustrations upon this subject show quite correctly the mucous membrane loosely separated outside the speculum, whereas a sub-muco-perichondrium could not be so illustrated.

It is better to imagine the cartilage of the septum to be suspended in a covering (Fig. 5), completely enclosed in front and only opening behind at the ethmoid margin, where it is anatomically continuous with its periosteum.

If this fact be grasped, it will be at once seen that any elevator once entering beneath this covering has no chance of exit except by force.

An elevator passed between the perichondrium and the cartilage and pressed downwards will find its way into the joint round the cartilage and up the opposite side. This fact can be readily proved by experiments.

The perichondrium itself is capable of being split into at least two different layers. Upon the lateral surface of the cartilage it is very 
firmly attached, and less so to the mucous membrane; but, once it reaches around the articular surface below the edge of the osseous groove, it is only loosely attached. The real periosteum rolls loosely over this bony edge, where it lines the bony trough (the sulcus vomeralis) and joins the opposite layer. If the cartilage is pushed well over to eitber side, the condition of the attachment at one time loose and another time tight, is readily seen. A layer of fat is met with between the perichondrium and periosteum, and also between the periosteum and floor of the vomeral groove.

If the cartilage removed from a recent septum be examined it will appear to be destitute of covering, but an incision into it and firm

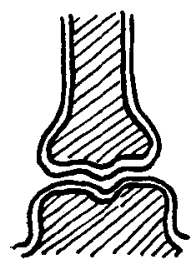

Fig. 8.



Fig. 9.

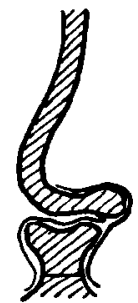

Fig. 10.

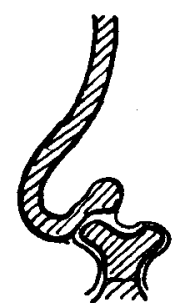

Fig. 11.

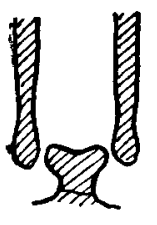

Fig. 12.

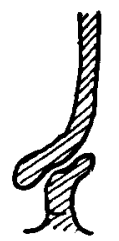

Fig. 13.



Fig. 14.

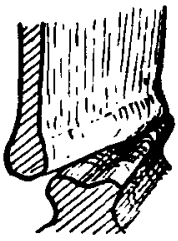

Fig. 15.

Fia. 8.-Section of chondro-maxillary joint showing the arrangement of the perichondrium and periosteum.

Fig. 9.-Another diagrammatic section showing the included layer of fat, the capsule and mucous membrane (on one side).

Fig. 10.-Dislocation of septal cartilage presenting a condition frequently mistaken for a spur of bone.

Figs. 11, 12, 13, 14.-Types of dislocation at the chondro-vomeral joint.

Fig. 15.-Type of chondro-maxillary displacements.

pressure with an elevator will reveal the perichondrium intact. In some instances the perichondrium may be found to have been removed for a short distance on the proximal side of the cartilage, but seldom, if ever, on the distal one.

I am not advocating any attempt to remove the perichondrium intact. I think it is very questionable whether two intact surfaces of perichondrium left behind would serve any useful purpose, much less if they were in a bruised and torn condition.

\section{The Membranous Folds of the Chondro-Voneral Join't.}

When passing the elevator downwards, it is a golden rule, upon approaching the joint, to stretch the parts well outwards, or, where considerable deformity exists, to cut the membrane from behind 
forwards and towards the cartilage with a curved blunt-ended bistoury. In most cases, if the elevator is pressed firmly outwards at this point and force used, the attachments will give way and the elevator pass downwards between the bone and the periosteum.

It must be remembered that; if the cartilage is (for example) leaning over to the left, the attachments on this side will be loose, and it is quite easy to force the elevator, membrane and all, into the joint. It is a good plan, therefore, when dividing the same with a bistoury, to press the cartilage well over to the opposite side to that on which the incision will be made, for by so doing the membranes attached are put upon the stretch.

The membranous folds of the chondro-maxillary joint are for all practical purposes similar, but (as before describèd) thicker, looser, and tougher.

\section{Fractures and Dislocations of the Septal Cartilage.}

Although acute conditions of the above are frequently referred to in the text-books, their nature is seldom defined, for very obvious reasons, whereas the chronic conditions of luxation and dislocation are extensively dealt with. Fig. 10 is by no means an uncommon condition; Fig. 11 rare; while Fig. 12 shows a direct dislocation either side of the sulcus vomeralis. I have never seen such a dislocation. The loose condition of the joint coverings allows for extensive displacement, but long before this direct lateral dislocation could take place the cartilage would probably double up. Further, unless the nose be crushed in or the cartilage be torn from the under surface of the nasal bones and the whole margin of the ethmoidal, any sudden dislocation is a mechanical improbability. In Figs. 13 and 14 we notice quite common forms due to the absence of the rim of the sulcus. Fig. 15 is not an uncommon form. The main portion of the cartilage is retained in the vomeral joint, but that over the crista incisiva is dislocated diagonally.

With regard to fractures, I am far more inclined to look to the upper and posterior borders for possible trouble, because they are not provided with movable joints. Sufficient force applied to the anterior portion of the nose must do one of two things-either fracture and dislocate the nasal bones, or depress, dislocate, and tear the upper grooved edge of the cartilage from the under surface of the nasal bones, and in this case it will probably break it off from the lamina ethmoidalis perpendicularis, and thereby deflect the anterior aspect of the nose. In this way only can I account for the fact of a crooked nose without displacement of the nasal bones after an accident.

\section{The Chondro-vascular System.}

Situated in an irregular line (Fig. 1, vii) will be seen the orifices and branching of the canals forming this complete system. These canals occupy the whole area described as the pear-shaped lower border, and also the portion anterior to this. They contain blood-vessels which arise from the mucous membrane and penetrate the perichondrium. These canals do not in any case communicate with each other, the vessels entering and returning by the same orifice. This vascular system is best studied and demonstrated in the septal cartilage of the sheep, when the septum, devoid of perichondrium, has become nearly dry. It may 
be beautifully seen by holding the cartilage before a strong light against a "bright white background.' In woll-developed fresh human subjects the same arrangement can easily be recognised. These vascular canals are very characteristic and distinctive; they never anastomose, they present rounded ends, and seldom, when branched, project beyond the centre of the cartilage. Upon the other hand, single straight canals can frequently be seen passing across the upper portions of the cartilage.

Since writing this article my work as a plastic surgeon at the Cambridge Hospital, Aldershot, has given me an unique opportunity of examining a large number of costal cartilages, and I have no hesitation in saying that they possess a vascutar system precisely similar to that described above in the septal cartilage. The system is very plain to the naked eye, and can be easily traced by the presence of blood. I therefore venture to disagree with those anatomists who describe such a condition as mere prolongations of persistent perichondral processes. The only difference I have so far been able to find between the vascular canals in fœtal cartilage and the vascular canals in the permanent cartilages referred to, is that in the former the ends of the canals are more pointed and branched, whilst in the latter they are rounded. Injections of these canals produce very interesting conditions, but their description I must leave to another occasion.

\section{Surgical Importance.}

If the coverings of the cartilage are removed upon both sides at the chondro-maxillary joint, the complete internal blood-supply of this thickened piece of cartilage will be found destroyed, a fact which leads to the question as to what eventually becomes of this pillar base which is left by the surgeon for cosmetic purposes.

I have very great pleasure in acknowledging valuable assistance from Prof. Wood Jones, who very kindly prepared illustrations from my rough drawing, and from Dr. Watkins. Pitchford of the South African Institute for Medical Research, Johannesburg.

\section{CLINICAL NOTE.}

\section{OBLITERATION OF THE CAVITY OF THE ANTRUM AFTER NASAL. ANTROSTOMY.}

\section{By Dan MCKenzIE.}

NASAL antrostomy-the formation of an opening into the nasal wall of the antrum through the nose-is still on trial, and one of the objections raised to the operation, admittedly partial, is that as it does not enable us to see to the complete removal of the lining of the cavity, the chances of curing the disease are but remote. Again, several years ago there was considerable discussion on the subject of the obliteration of the frontal and other nasal sinuses by fibrous tissue as a result of operation, and it was pointed out at the time and generally agreed to that this desirable. result was most probable when the lining of the sinus was cleared out.

I do not for a moment doubt that this, as a rule, is probably correct. But the same agreeable change may follow simple nasal antrostomy, as I have recently found. 